\title{
Praticare l'appropriatezza in Medicina di Laboratorio. Un aggiornamento
}

\author{
Practicing appropriateness in Laboratory Medicine. An update
}

\author{
Piero Cappelletti ${ }^{1}$
}

Ricevuto: 16 marzo 2016 / Accettato: 11 aprile 2016 / Pubblicato online: 9 giugno 2016

(C) Società Italiana di Patologia Clinica e Medicina di Laboratorio 2016

Riassunto In Medicina di Laboratorio, l'evoluzione del concetto di appropriatezza e le più recenti informazioni di letteratura confermano il ruolo dell'EBLM (evidence-based laboratory medicine) nel fondarla e l'aderenza alle linee guida "evidence-based" come criterio da seguire, consapevoli che l'inappropriatezza per difetto è dimostratamente superiore a quella per eccesso. Il concetto della necessità di declinare l'appropriatezza per ogni passaggio del TTP (total testing process) è oggi accettato sulle due sponde dell'Atlantico, tuttavia l'attenzione è ancora principalmente volta alla selezione dei test. Da questo punto di vista, esperienze anche italiane mostrano il ruolo importante degli strumenti informatici di supporto alla decisione clinica e dell'iniziativa Choosing Wisely. Le evidenze di letteratura sono tali da consentire il passaggio dalla medicina basata sulle prove di efficacia a una politica sanitaria condotta e supportata dalle "evidenze" e dalle linee guida professionali su di esse costruite.

Parole chiave Appropriatezza - Evidence-based laboratory medicine $\cdot$ Linee guida $\cdot$ Choosing Wisely

Summary The evolution of the concept of "appropriateness" and some recent reports confirm the role of Evidencebased Laboratory Medicine in its foundation and the adherence to evidence-based guidelines as reference source of the criteria for tests appropriateness. It implies that appropriateness of tests could be not only overuse but also underuse and a recent meta-analysis shows that the last one is double than the first one in Laboratory Medicine. Moreover, the concept of appropriateness as a quality of every phase of

\footnotetext{
$凶$ P. Cappelletti

pie.cappelletti@gmail.com

1 SIPMeL, Castelfranco Veneto, TV, Italia
}

the Total Testing Process has been accepted both theoretically and practically. Nevertheless, the large majority of reports about appropriateness are still dedicated to the selection of tests. From this point of view, many experiences, also in Italy, show the crucial role of Computerized Clinical Decision Support Systems and of the Choosing Wisely Initiative. The data from recent literature allow the substantial shift from evidence-based laboratory medicine to evidencedriven health management and public policy. According to Matthew McQueen, the ultimate challenge may be to recognize openly the political uses and misuses of evidence and to extend rules of evidence-based appropriateness to government policy.

Keywords Appropriateness - Evidence-based laboratory medicine · Guidelines · Choosing Wisely

\section{Introduzione}

Qualche anno fa, due Editoriali dedicati all'appropriatezza hanno chiarito che praticare l'appropriatezza in Medicina di Laboratorio è complesso, perché il concetto è elusivo anche se è andato maturando nel tempo, perché non sempre sono "evidenti" o robusti i fondamenti EBLM su cui si dovrebbero costruire i criteri [1] e perché gli strumenti d'implementazione sono multifattoriali e richiedono continuità e attenta verifica, e, inoltre, hanno suggerito una sorta di vademecum (Tab. 1) [2]. Recentemente la letteratura ha offerto nuovi elementi interessanti, in relazione ai fondamenti evidence-based dell'appropriatezza, al concetto di appropriatezza declinato per tutto il processo diagnostico (TTP) e agli strumenti di implementazione pratica, a partire dalle definizioni più recenti di appropriatezza di un esame diagnostico come ciò che fornisce una risposta al quesito clinico e 
Tabella 1 Vademecum per l'implementazione di percorsi di appropriatezza in Medicina di Laboratorio

1 Scegliere un gruppo multidisciplinare e multiprofessionale di lavoro all'interfaccia clinica-laboratorio, riconosciuto dall'alta direzione, per trovare o produrre evidenze e linee guida e condurre regolari feedback e/o audit

2 Gli obiettivi devono essere rilevanti ma raggiungibili; l'appropriatezza si costruisce per mattoni singoli

3 Condividere con i clinici scelte e pratiche riferite a obiettivi, linee guida, strumenti d'implementazione e metodi di verifica continuativa

4 Il leader del laboratorio, con l'equipe multiprofessionale e multidisciplinare di laboratorio, sceglie dispositivi, metodi, formato dei risultati, percorsi interni di appropriatezza e modi di interpretazione/consulenza per produrre il massimo di informazioni diagnostiche efficaci

5 Lavorare alacremente nella diffusione dell'educazione, nell'implementazione degli strumenti di appropriatezza e nel rinforzo continuo delle linee d'azione: l'appropriatezza è attività trasformativa

quindi consente una decisione a cui consegue un trattamento [3] e che è misurata come informazione che determina esiti rilevanti per il paziente [4].

\section{Appropriatezza ed EBLM}

Il legame tra appropriatezza ed esiti clinici, messo in risalto dalle definizioni appena ricordate e legato al cambiamento da "essenzialista" a "consequenzialista" della Medicina di Laboratorio [5], implica che il fondamento dell'appropriatezza in Medicina di Laboratorio sia l'EBLM, evitando il rischio di soggettività messo in luce da Smellie nel suo Appropriateness of test use in pathology: a new era or reinventing the wheel? [6].

Il criterio di riferimento per l'appropriatezza diventa, quindi, l'aderenza alle linee guida. Nella ricerca del 2014 di Hauser e Shirts [7], 1'84,5\% dei criteri di appropriatezza riposavano, appunto, su linee guida clinico-organizzative nazionali (governo, Società scientifiche), locali o da letteratura.

Un'immediata conseguenza è che l'inappropriatezza non è solo eccesso di test, come superficialmente dedotto dal lavoro di van Walrawen e Naylor [8], ma anche sottoutilizzo o errata interpretazione dei risultati [5]. Da questo punto di vista i pochi lavori disponibili in letteratura sono stati irrobustiti dalla metanalisi sulle ricerche dei 15 anni (1997-2012) successivi alla ricerca di van Walraven e Naylor, eseguita da Zhi et al [9], che mostra un tasso medio di sovrautilizzo del $20,6 \%$ (IC 95\% 16,2-24,9\%), ma un tasso medio di sottoutilizzo del 44,8\% (IC 95\% 33,8-55,8\%). Il valore cumulativo d'inappropriatezza si situa, dunque, intorno a quel $33 \%$ stimato in precedenza, ma per effetto di un sottoutilizzo doppio del sovrautilizzo.

È tuttavia noto che le evidenze in Medicina di Laboratorio sono meno forti che in campo terapeutico, per la carenza di studi primari e la debolezza delle ricerche sistematiche e delle metanalisi, per le ragioni elencate da Bruns [10] ancora nel 2001. Per questo il Report 2009 del Lewin Group [11] ha sancito che gli studi clinici randomizzati (RCT) non dovrebbero essere utilizzati nelle ricerche di efficacia comparata in
Medicina di Laboratorio. Il problema chiave è la distanza spazio-temporale tra esame diagnostico ed esiti nel decorso della malattia, pertanto si suggerisce di utilizzare studi di accuratezza diagnostica secondo i recentemente rinnovati standard STARD [12] e QUADAS [13] oppure si è costretti a utilizzare ancora processi di consenso come "gruppi nominali", "consensus conference" o metodi "Delphi” più o meno semplificati [1].

Nonostante ciò, le linee guida non riescono a coprire tutta la realtà operativa. Per l'ematologia di laboratorio, per esempio, nel 2006 [14] le 88 evidenze dell'EBLM-Commission di IFCC erano relative alla coagulazione (53\%), $22 \%$ ai leucociti (di cui metà per sepsi e metà per sottopopolazioni linfocitarie), $19 \%$ a eritrociti ed emoglobina e $6 \%$ a piastrine e nel 2015 [15] le 64 linee guida per l'ematologia di laboratorio in PubMed e altri siti davano risultati simili: $40 \%$ coagulazione, $26,4 \%$ leucociti (circa $60 \%$ per citofluorimetria), $14,7 \%$ per emoglobinopatie e altre anomalie eritrocitarie e $5,9 \%$ piastrine. Produrre evidenze e linee guida è, dunque, un campo necessario ed eccitante per la Medicina di Laboratorio [16], tenendo conto che poco più del $20 \%$ delle linee guida generali comprende nel suo gruppo di ricerca anche un professionista di laboratorio [7].

\section{Appropriatezza e TTP}

Più di dieci anni fa il GdS E SIMeL [14] affermava che l'appropriatezza è un predicato di ogni fase del TTP, se si vuol essere conseguenti alle definizioni di appropriatezza prima ricordate. Oggi pare che il concetto sia stato largamente accettato sia teoreticamente sia praticamente.

La Task Force IFCC sull'impatto della Medicina di Laboratorio sulla gestione clinica e sugli esiti [17], citando le cinque vie con cui può avverarsi un errore diagnostico [18], esplicita la necessità di controllare tutti i passaggi del TTP. D'altro canto Fryer e Smellie [19], nel loro "toolkit" per l'appropriatezza degli esami di laboratorio, suggeriscono 27 raccomandazioni non solo focalizzate alla selezione dei test ma anche alla fase pre-preanalitica (condividere la 
strategia con i clinici) e preanalitica (prelievo e caratteristiche del campione), postanalitica (referto interpretativo) e post-postanalitica (audit), con particolare enfasi sull'impatto delle richieste computerizzate e delle esigenze dei pazienti.

Ciò apre un ulteriore campo all'attività dei professionisti del laboratorio [16]. Le linee guida cliniche non trattano di solito gli aspetti considerati laboratoristici: solo in un terzo dei casi sono presenti le necessarie informazioni riguardo lo stato dei pazienti, le interferenze biologiche e analitiche e il trattamento dei campioni [7]. L'inserimento di uno specialista di laboratorio nei comitati/gruppi di preparazione di linee guida cliniche, però, migliora la situazione, rendendole esaustive sotto il profilo dei mezzi diagnostici in circa la metà dei casi [7].

Tuttavia, la gran parte della letteratura sull'appropriatezza è ancora dedicata alla selezione dei test, sotto il nome di "laboratory utilization management" o più precisamente "demand management" se focalizzata all'appropriatezza e "demand control" se riferita al contenimento del numero delle richieste [20]. Buone revisioni di letteratura, dal punto di vista anglosassone e americano, sono comparse a cura di Smellie [21] e di Huck e Lewandroski [22].

\section{Praticare l'appropriatezza}

Le conclusioni di Solomon et al [23] sui modi di implementare regole di appropriatezza sono state ancora recentemente confermate da una revisione sistematica su 109 lavori selezionati dai 3236 raccolti nell'arco degli anni 1946-2013 [24]: la strategia migliore è multifattoriale e prolungata e comprende un intelligente mix di educazione, cambiamenti amministrativi del disegno/modo di richiesta e feedback e/o audit. Inoltre il supporto degli opinion leader nei gruppi clinici e dell'alta dirigenza ai gruppi multidisciplinari è cruciale per il successo delle iniziative [20]. Tuttavia la qualità dei lavori di letteratura è bassa e l'eterogeneità è molto elevata, quindi i suggerimenti devono essere cercati nelle singole esperienze [24].

Tra le diverse strategie proposte per modificare il comportamento dei clinici, negli anni recenti due novità hanno fatto intravvedere positive possibilità di successo: i CCDSS (Computerized Clinical Decision Support Systems), di cui abbiamo già parlato [2], e l'iniziativa Choosing Wisely [25].

Per quanto riguarda i primi si vanno accumulando dati sull'efficacia dei sistemi che allertano o bloccano i richiedenti in base a regole di contesto o di ripetizione. Secondo recenti dati americani [26], i sistemi di blocco sono più efficienti di quelli di allerta, almeno per quel che riguarda le richieste duplicate $(92,3 \%$ contro $42,6 \%)$. Più articolata la situazione italiana. Il Progetto ERMETE della Regione Veneto, ora all'esame del Ministero della salute, si basa sul GOELM DSS (Guide Order Entry Laboratory MedicineDecision Support System), un modello a razionalità limitata che propone alcune opzioni e suggerisce alcune indicazioni per la richiesta dei test, ma non impone o blocca scelte. Nel 2010 due esperimenti con complessivi 219 medici di medicina generale (MMG) su 110 test hanno raggiunto riduzioni cumulative del 26\% e 38\% rispettivamente [27]. Il Progetto AdeR dell'Area Vasta Romagna utilizza un software di 128 regole che consente o blocca la richiesta di test ed è stato testato dal 2010 in 7 ospedali su 18 esami selezionati (AST/ALT; urea/creatinina; VES/CRP; marcatori tumorali; $\mathrm{HbA}_{1 \mathrm{c}}$; elettroforesi proteica). Nel 2015 la richiesta per i test sopra ricordati è diminuita tra il $45,4 \%$ e il $93,9 \%$ con l'unica crescita della CRP $(+38 \%)$ contro una diminuzione della VES del 93,9\% [28]. L'implementazione dei due sistemi è stata preceduta da un'intensa attività educativa $\mathrm{e}$ accompagnata da regolari feedback. Al di là delle differenze nella tipologia (MMG vs medici ospedalieri) e nel numero di medici coinvolti $(219 v s \approx 1000$ ), nel numero dei test selezionati (110 vs 18) e nelle caratteristiche degli strumenti (allerta $v s$ blocco), entrambi i sistemi dimostrano che il mix di fattori predisponenti, abilitanti e rinforzanti funziona.

Choosing Wisely [25] è un'iniziativa lanciata nel 2012 da ABIM (American Board of Internal Medicine) con l'obiettivo di evitare test, trattamenti e procedure inutili sulla base di raccomandazioni espresse dalle Società scientifiche in maniera sintetica "The Top Five List" (le 5 cose da non fare), una lista di cinque esami diagnostici o trattamenti che abbiano come caratteristiche di essere tra i più costosi, di esporre i pazienti a rischi e non apportare benefici significativi. L'iniziativa si è diffusa nel mondo e la sezione italiana Choosing Wisely_Slow Medicine [29] comprende ora più di 40 Società scientifiche, inclusa SIPMeL, e più di 10 organizzazioni professionali. I due aspetti salienti dell'iniziativa sono, dal lato professionale, il suggerimento di raccomandazioni veloci e facilmente memorizzabili, piuttosto che le lunghe e complicate linee guida spesso aggiornate e riaggiornate, che sono molto più gradite alla maggior parte dei medici e, dal lato dei pazienti, il concorso nell'implementazione di coalizioni di associazioni di pazienti e consumatori che ne garantiscono la diffusione e l'accettazione, superando la percezione di ingiustificati razionamenti di cure [19]. La SIPMeL ha presentato una lista generale (Tab. 2) e il GdS Endocrinologia e Malattie del Metabolismo, per primo, ha preparato un' altra specifica lista che si riferisce alla diagnostica ottimale della funzionalità tiroidea, della sindrome di Cushing, del feomocromocitoma, di tireopatia autoimmune e alla determinazione della $25(\mathrm{OH})$ vitamina $\mathrm{D}[30]$.

\section{Conclusioni}

In un periodo storico contrassegnato dalla necessità del contenimento dei costi ma anche dall'esigenza di un'attenzione maggiore alla qualità ed efficacia delle cure [31], spesso 
Tabella 2 "Top five list" di SIPMeL per Choosing Wisely_Slow Medicine

\begin{tabular}{|c|c|}
\hline 1 & NON richiedere CK-MB, CK totale, AST, LDH e MIOGLOBINA nella diagnostica di sindrome coronarica acuta (SCA) \\
\hline 2 & NON richiedere la determinazione dell'esame GLICOSURIA nel monitoraggio di un paziente con diabete mellito \\
\hline 3 & $\begin{array}{l}\text { NON richiedere esami di laboratorio preoperatori in pazienti a basso rischio (per chirurgia minore e per chirurgia intermedia nei pazienti } \\
\text { ASA } 1 \text {, ASA } 2 \text { se non AKD, ASA } 3 \text { e } 4 \text { in pazienti senza malattia cardiovascolare, renale e diabetica) }\end{array}$ \\
\hline 4 & $\begin{array}{l}\text { NON richiedere elettroforesi proteica e la ricerca della proteina di Bence Jones urinaria come esami di idoneità per l'esecuzione di esami } \\
\text { radiologici con mezzi di contrasto }\end{array}$ \\
\hline 5 & NON richiedere i cosiddetti "marcatori tumorali" Ca 125, Ca19-9, Ca 15-3, HE4, CEA e alfa-fetoproteina in pazienti asintomatici \\
\hline
\end{tabular}

le scelte di "appropriatezza" si risolvono in interventi di razionamento, calati dall'alto, che possono sì avere effetti finanziari, ma aprono molti dubbi circa l'appropriatezza vera delle cure, lasciando possibile campo al sottoutilizzo di trattamenti efficaci [32]. Pur non negando la necessità di maggiore attenzione ai modi concreti di attuare il principio "less is more", una riflessione collettiva (professionisti, decisori e stakeholders in primis, pazienti e cittadini) sulle "evidenze" di letteratura, sui modi già testati anche in Italia di praticare l'appropriatezza e sulle capacità della Società scientifiche come SIPMeL di individuare linee guida corrette ed efficaci dovrebbe essere il viatico necessario per qualsiasi intervento locale e nazionale. Sarebbe ora che dalla "evidence-based medicine" si raggiungesse quella che Matthew McQueen [33] chiamava già 15 anni fa "evidencedriven health management and public policy": "The ultimate challenge may be to recognize openly the political uses and misuses of evidence and to extend rules of evidence to government policy".

\section{Conflitti di interesse Nessuno.}

Studi condotti su esseri umani e animali Non applicabile.

Consenso informato Non applicabile.

\section{Bibliografia}

1. Cappelletti P (2013) Praticare l'appropriatezza in Medicina di Laboratorio. Una introduzione. Riv Ital Med Lab 9:1-7

2. Cappelletti P (2013) Praticare l'appropriatezza in Medicina di Laboratorio. Istruzioni per l'uso. Riv Ital Med Lab 9:55-60

3. Price CP (2003) Application of the principles of evidence-based medicine to laboratory medicine. Clin Chim Acta 333:147-154

4. Trikalinos TA, Siebert U, Lau J (2009) Decision-analytic modeling to evaluate benefits and harms of medical tests-uses and limitations. Agency for Healthcare Research and Quality, Rockville

5. Bossuyt PM (2012) The architecture of medical test evaluation. http://assr.regione.emilia-romagna.it/it/eventi/2012/to-see-or-notto-see/Bossuyt_architecture.pdf/at_download/file/Bossuyt_ architecture.pdf (Accesso 16 marzo 2016)

6. Smellie WSA (2003) Appropriateness of test use in pathology: a new era or reinventing the wheel? Ann Clin Biochem 40:585592
7. Hauser RG, Shirts BH (2014) Do we now know what inappropriate laboratory utilization is? An expanded systematic review of laboratory clinical audits. Am J Clin Pathol 141:774-783

8. van Walraven C, Naylor CD (1998) Do we know what inappropriate Laboratory utilization is? JAMA 280:550-558

9. Zhi M, Ding EL, Theisen-Toupal J et al (2013) The landscape of inappropriate laboratory testing: a 15-year meta-analysis. PLoS ONE 8:e78962

10. Bruns DE (2001) Laboratory-related outcomes in Healthcare. Clin Chem 47:1547-1552

11. The Lewin Group (2009) The value of laboratory screening and diagnostic tests for prevention and health care improvement. http:// thehill.com/sites/default/files/acla_lewinvalueoflabsxdxreport_0. pdf (Accesso 16 marzo 2016)

12. Bossuyt PM, Reitsma JB, Bruns DE et al (2015) STARD 2015: an updated list of essential items for reporting diagnostic accuracy studies. BMJ 351:h5527

13. Whiting PF, Rutjes AW, Westwood ME et al (2011) QUADAS-2: a revised tool for the quality assessment of diagnostic accuracy studies. Ann Intern Med 155:529-536

14. Cappelletti P (2006) Appropriatezza in Ematologia di Laboratorio. RIMeL-IJLaM 2:119-129

15. Hayward CPM, Moffat KA, George T et al (2015) Assembly and evaluation of an inventory of guidelines that are available to support clinical hematology laboratory practice. Int J Lab Hematol 37:36-45

16. Cappelletti P (2015) Linee guida e SIPMeL. Riv Ital Med Lab 11:185-190

17. Hallworth MJ, Epner PL, Ebert C et al (2015) Current evidence and future perspectives on the effective practice of Patient-Centered Laboratory Medicine. Clin Chem 61:589-599

18. Epner PL, Gans JE, Graber ML (2013) When diagnostic testing leads to harm: a new outcomes-based approach for laboratory medicine. BMJ Qual Saf 22(Suppl 2):ii6-ii10

19. Fryer AA, Smellie WSA (2013) Managing demand for laboratory tests: a laboratory toolkit. J Clin Pathol 66:62-72

20. Yeh DD (2014) A clinician's perspective on laboratory utilization management. Clin Chim Acta 427:145-150

21. Smellie WSA (2012) Demand management and test request rationalization. Ann Clin Biochem 49:323-336

22. Huck A, Lewandroski K (2014) Utilization management in the clinical laboratory: an introduction and overview of the literature. Clin Chim Acta 427:111-117

23. Solomon DH, Hashimoto H, Daltroy L et al (1998) Techniques to improve physicians' use of diagnostic tests. A new conceptual framework. JAMA 280:2020-2027

24. Kobewka DM, Ronksley PE, McKay JA et al (2015) Influence of educational, audit and feedback, system based, and incentive and penalty interventions to reduce laboratory utilization: a systematic review. Clin Chem Lab Med 53:157-183 
25. http://www.choosingwisely.org/about-us/ (Accesso 16 marzo 2016)

26. Procop GW, Keating C, Stagno P et al (2015) Reducing duplicate testing. A comparison of two clinical decision support tools. Am J Clin Pathol 143:623-626

27. Camerotto A, Truppo V, Bedendo S et al (2014) ERMETE, il progetto della Regione Veneto per la gestione della conoscenza nella Medicina di Laboratorio. Riv Ital Med Lab 10:125-135

28. Morotti L, Vascotto L, Meluzzi D et al (2015) Valutazione efficacia dell'applicazione regole di appropriatezza. $1^{\circ}$ Congresso Nazionale Società Italiana di Patologia Clinica e Medicina di Laboratorio, Roma 24-26 novembre
29. http://www.slowmedicine.it/i-progetti/fare-di-piu-non-significafare-meglio.html (Accesso 16 marzo 2016)

30. Dorizzi RM, Ferrari A, Vitillo M et al (2016) Choosing Wisely: la lista del gruppo di studio. Endocrinologia e Malattie del Metabolismo della Società Italiana di Patologia Clinica e Medicina di Laboratorio Riv Ital Med Lab. doi:10.1007/s13631-016-0115-9

31. Cappelletti P (2012) Il futuro della Sanità: sostenibilità finanziaria e scenari evolutivi. Riv Ital Med Lab 8:63-70

32. http://www.slowmedicine.it/notizie/143-appropriatezza-prescrittiva/ 424-decreto-appropriatezza.html (Accesso 16 marzo 2016)

33. McQueen MJ (2001) Overview of evidence-based medicine: challenges for evidence-based Laboratory Medicine. Clin Chem 47:1536-1546 